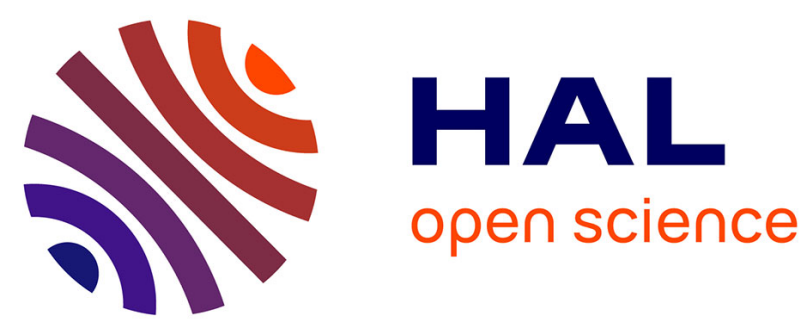

\title{
PROTON AND PROTON-COUPLED ELECTRON TRANSFER WITH PARADIGMS TOWARDS SINGLE-MOLECULE SYSTEMS
}

Jens Ulstrup, Alexander Mikhailovitch Kuznetsov

\section{- To cite this version:}

Jens Ulstrup, Alexander Mikhailovitch Kuznetsov. PROTON AND PROTON-COUPLED ELECTRON TRANSFER WITH PARADIGMS TOWARDS SINGLE-MOLECULE SYSTEMS. Journal of Physical Organic Chemistry, 2010, 23 (7), pp.647. 10.1002/poc.1724 . hal-00552421

\section{HAL Id: hal-00552421 \\ https://hal.science/hal-00552421}

Submitted on 6 Jan 2011

HAL is a multi-disciplinary open access archive for the deposit and dissemination of scientific research documents, whether they are published or not. The documents may come from teaching and research institutions in France or abroad, or from public or private research centers.
L'archive ouverte pluridisciplinaire HAL, est destinée au dépôt et à la diffusion de documents scientifiques de niveau recherche, publiés ou non, émanant des établissements d'enseignement et de recherche français ou étrangers, des laboratoires publics ou privés. 


\section{PROTON AND PROTON-COUPLED ELECTRON TRANSFER WITH PARADIGMS TOWARDS SINGLE-MOLECULE SYSTEMS}

\begin{tabular}{|c|c|}
\hline Journal: & Journal of Physical Organic Chemistry \\
\hline Manuscript ID: & POC-10-0005.R1 \\
\hline Wiley - Manuscript type: & Review Commentary \\
\hline $\begin{array}{l}\text { Date Submitted by the } \\
\text { Author: }\end{array}$ & 22-Mar-2010 \\
\hline Complete List of Authors: & $\begin{array}{l}\text { Ulstrup, jens; Technical University of Denmark, Chemistry } \\
\text { Kuznetsov, Alexander; Russian Academy of Sciences, The A.N. } \\
\text { Frumkin Institute of Physical Chemistry and Electrochemistry }\end{array}$ \\
\hline Keywords: & $\begin{array}{l}\text { Proton conduction, Hydrogen atom transfer, Single-molecule proton } \\
\text { transfer }\end{array}$ \\
\hline
\end{tabular}

\section{今 scholaronE" \\ Manuscript Central}




\title{
PROTON AND PROTON-COUPLED ELECTRON TRANSFER WITH PARADIGMS TOWARDS SINGLE-MOLECULE SYSTEMS
}

\author{
A.M. Kuznetsov ${ }^{1}$ and J. Ulstrup ${ }^{2}$ \\ ${ }^{1}$ The A.N. Frumkin Institute of Physical Chemistry and Electrochemistry of the \\ Russian Academy of sciences \\ Leninskij Prospect 31 \\ 119993 Moscow, Russia \\ ${ }^{2}$ Department of Chemistry \\ Building 207, Technical University of Denmark \\ Kemitorvet \\ DK-2800 Kongens Lyngby \\ Denmark
}

\begin{abstract}
Proton (PT), H-atom, and proton coupled electron transfer (PCET) are ubiquitously encountered in chemical and biological processes. PT and H-atom transfer can belong to the partially or totally adiabatic limits representing "weak" or "strong" interactions between the donor and acceptor, reflected most conspicuously in large, i.e. $>>1$ and small, i.e. 1-2, kinetic deuterium isotope effects (KIE). In view of the short proton/H-atom transfer distances the electronically adiabatic limit prevails in either case. The PCET notion applies from sequential PT and ET events to fully synchronous ET/PT as in H-atom transfer.

We overview first these notions. We then address several classes of PT reactions not commonly addressed in analytical condensed matter PT/H-atom transfer theory. These include viscosity (relaxation) controlled PT and KIE $<1$ in protein systems. Other classes are PT in strongly hydrogen bonded systems such as excess proton conduction in aqueous solution or in biological or synthetic membranes, and PT in the "inverted" free energy region where excited proton vibrational states and a maximum in the Brønsted relation are important.

We finally invoke an approach to single-molecule PT and $\mathrm{H}$-atom transfer where the PT/H-atom transferring molecules are enclosed between the substrate and tip in electrochemical (in situ) scanning tunneling microscopy (STM) or between a pair of nanoscale electrodes. No data are
\end{abstract}




\section{Introduction}

The ubiquity of proton transfer (PT) processes in chemistry and biology is equalled only by electron transfer (ET). These two "elementary" reaction classes in condensed matter environment were previously addressed quite differently. PT was regarded as essentially classical proton motion in a double-well potential spanned by the proton stretching mode, with vibrational zero-point behaviour near the bottom of the reactants' well and proton tunneling "corrections" near the barrier top ${ }^{1-3}$. ET was regarded as a quantum mechanical transition between the electron donor and acceptor molecular entities induced by intermolecular interactions combined with environmental and local mode configurational fluctuations ${ }^{4,5}$.

A radically novel view of PT reactions was introduced from the late 1960s by Dogonadze, Kuznetsov and their associates ${ }^{4-8}$. Classical nuclear motion and activation (free) energy was noted to be determined entirely by low-frequency solvent and other heavy nuclear motion. Protons were instead naturally represented by quantum mechanical tunneling not only near the barrier top of the proton part of the potential surfaces but in general. This view of vibrationally assisted proton tunneling is the present day prevalent view and the basis for most later approaches to condensed matter PT and H-atom transfer processes ${ }^{9-14}$, even though close to classical PT behaviour is expected in strongly $\mathrm{H}$-bonded systems ${ }^{10,15}$. This view of tunneling along high-frequency PT stretching (or bending) modes is paralleled by nuclear tunneling in other local high-frequency modes in ET processes ${ }^{4,5,16}$.

PT viewed as a quantum mechanical transition discloses both close physical and formal analogies with ET processes and some differences. In either case light particles are transferred by tunneling between much heavier donor and acceptor fragments. By their localized electrostatic charges both particles are also strongly coupled to the (polar) solvent environment. An important difference is that PT is a more composite "elementary" reaction than ET by involving synchronous bond breaking and formation, while the donor and acceptor groups in an elementary ET step retain their structural integrity. Another difference is that electron tunneling over "long" distances, i.e. up to several nanometers is feasible, whereas by the much heavier proton mass, PT is only possible over less than an Ångström ${ }^{17,18}$. "Long-range" PT such as in proton conduction channels in large protein 
complexes, say cytochrome $c$ oxidase or-22 $^{19}$ or through the water solvation sphere in hydrolytic enzyme reactions, e.g. carbonic anhydrase ${ }^{23-25}$, must therefore involve a sequence of much shorter vibrationally assisted proton tunneling steps ("hops"). Each of these must further be "gated" by environmental nuclear mode fluctuations as the equilibrium proton donor-acceptor distance even of each of these steps is far too wide for proton tunneling. These expectations apply widely, for example also to proton conductivity in Nafion ${ }^{\circledR}$ and other synthetic membranes ${ }^{26}$.

Proton coupled ET, PCET in which PT and ET are to variable extent "coupled" have become key notions in a wide range of chemical and biological processes ${ }^{11,15}$. PCET are integrated parts of redox enzyme processes involving, for example conversion of dioxygen to hydrogen peroxide or water catalyzed by metallo-oxidases, or in membrane proton pumping such as in cyt $c$ oxidase. Excited state radical processes as in photoreactions of the green fluorescent protein ${ }^{27,28}$, radical enzyme mechanisms, photosynthesis, and elementary reaction steps in electrochemical dihydrogen evolution are other cases. The PCET notions imply that an ET step "triggers" a PT step or vice versa. Four states, instead of $\boldsymbol{t w o}$ as in separate single-ET or -PT are therefore involved in schemes such as ${ }^{15}$

$\begin{array}{ccccc} & \mathrm{D}_{\mathrm{e}} & \mathrm{A}_{\mathrm{e}} & \mathrm{D}_{\mathrm{p}} & \mathrm{A}_{\mathrm{p}} \\ \mathrm{i} & 1 & 0 & 1 & 0 \\ \mathrm{e} & 0 & 1 & 1 & 0 \\ \mathrm{p} & 1 & 0 & 0 & 1 \\ \mathrm{f} & 0 & 1 & 0 & 1\end{array}$

where 0 and 1 denote the occupation of the corresponding sites. $\mathrm{i}$ and $\mathrm{f}$ are the initial and final state, while $\boldsymbol{e}$ represents ET and $\boldsymbol{p}$ PT.

Two limiting cases can be recognized. One limit is entirely synchronous ET and PT between the same donor and acceptor sites. This limit reduces to hydrogen atom transfer as encountered in numerous chemical ${ }^{9,29,30}$ and biological ${ }^{9,11,13,14,27,28}$ radical reactions. These are often associated with large kinetic deuterium isotope effects (KIEs) ${ }^{9,12-14,29}$, caused by poor H-bonding between the donor and acceptor fragments and hampered translational motion of the two fragments to diminish the $\mathrm{H}$ - 
atom transfer distance. The other limit is completely independent ET and PT events, with full vibrational solvent or protein relaxation between the two steps. The two steps would here commonly involve different donor and acceptor sites, still close enough that, say ET at one site induces electrostatic field effects that affect $\mathrm{pK}_{\mathrm{a}}$ at the PT site, or vice versa, i.e. charge induced driving force effects. PT steps in cyt $c$ oxidase pumping triggered by ET from cyt $c$ to the $\mathrm{Cu}_{\mathrm{A}^{-}}$ centre $^{19-22}$ could be such an example. The two steps thus both involve full vibrational relaxation but are mutually dependent in the sense noted.

Partial environmental vibrational relaxation can be envisaged, but is better represented by transfer of two or several protons. The transfer of a given proton would initiate vibrational relaxation of both translational motion of the temporarily protonated first proton acceptor group and of water molecules in the environment. Before full vibrational relaxation has been reached the second proton is, however, passed on to the next acceptor molecule in the chain. Excess proton conduction in water (the Grotthuss mechanism) $)^{31-34}$ and double-PT in the serine protease triad ${ }^{35}$ are such examples, for which the notion "PT through dynamically populated intermediate states" has been suggested ${ }^{5,15}$.

PT and $\mathrm{H}$-atom transfer have been in long-time experimental and theoretical focus. Recent reviews are available $9,11-14,36,37$. We address here some concepts and theoretical notions of PT and H-atom transfer carried over to areas such as proton conductivity in strongly H-bonded systems ${ }^{31-33,38}$, the “inverted" free energy region ${ }^{39-41}$, solvent relaxation controlled $\mathrm{PT}^{42}$, cases of kinetic isotope effects less than unity ${ }^{43}$ and to novel approaches to single-molecule PT/H-atom transfer ${ }^{44}$.

\section{Partially and Totally Adiabatic Proton and Hydrogen Atom Transfer}

Large, i.e. $>10$ values of the KIE, indicative of strong quantum mechanical tunneling features have long been known for PT between poorly hydrogen bonded C-donors and -acceptors. Significantly larger values, i.e. up to two or even several orders of magnitude have been reported for $\mathrm{H}$-atom transfer in frozen glasses at low temperatures ${ }^{29}$ and in several enzyme processes at room temperature ${ }^{9,13,14}$. The large effects are associated with "freezing" of the proton gating mode by the solid matrix or by the protein framework. In most cases of PT between $\mathrm{O}$ - and $\mathrm{N}$-acids and -bases with strong hydrogen bonding along the PT mode, the KIEs are, however, small, i.e. weakly in excess of the stretching vibrational frequency ratio of $\sqrt{ } 2^{1,2,9,34}$. The equilibrium structural PT distance is, however, still significant, say $\approx 0.8 \AA$, indicative of strongly PT. The quantum mechanical nature of the transition is, however, maintained also in this limit, cf. below. 
The discussion above offers a PT scenario summarized in Figs. 1 and 2.. Extensive analysis is given in $^{12,34,37,45}$ from which we note:

Fig. 1

Fig. 2

- PT is represented by two nuclear mode sets with widely different time scales, viz. the proton mode $(s), r_{p}$, and the environmental solvent or protein conformational modes, $\left\{q_{\kappa}\left(r_{p}\right)\right.$. The proton is trapped at the donor in the reactants' equilibrium conformational/solvent configuration, but fluctuations in these modes induce resonance between the proton vibrational levels in the reactants' and products' states. Environmental gating is part of this process.

- Reorganization of the proton and electronic system parts proceeds in this dynamic state of resonance and is followed by trapping of the proton in the products' state.

- The electronic system part follows smoothly ("adiabatically") the nuclear reorganization in the proton resonance state but the proton motion may be obstructed by a tunneling barrier. This limit is denoted as the "partially adiabatic" limit. The proton may also follow "adiabatically" the environmental nuclear dynamics if the proton tunneling barrier is small. Attenuation of the proton tunneling barrier is part of the gating process as the barrier is narrower and shallower, the closer the gating mode has taken the donor and acceptor fragments towards each other.

- The partially adiabatic limit applies to PT involving C-acids and -bases and to hydrogen atom transfer, in redox enzymes or frozen glasses. The following rate constant forms apply ${ }^{4-8,12}$

$W_{R P}=Z_{P}^{-1} \sum_{v, w} \exp \left(-\frac{\varepsilon_{R v}^{P}}{k_{B} T}\right) W_{R P}^{v w}$

$W_{R P}^{v w}=\kappa_{v w}^{P} \frac{\omega_{e f f}}{2 \pi} \exp \left(-\frac{G_{v w}^{\neq}}{k_{B} T}\right) ; \quad Z_{P}=\sum_{v, w} \exp \left(-\frac{\varepsilon_{R v}^{P}}{k_{B} T}\right)$

$G_{v w}^{P}=\frac{\left[E_{r}+\Delta G^{0}+\left(\varepsilon_{P w}^{P}-\varepsilon_{P w=0}^{P}\right)-\left(\varepsilon_{R v}^{P}-\varepsilon_{R v=0}^{P}\right)\right]^{2}}{4 E_{r}}$ 


$$
\begin{aligned}
& \kappa_{v w}^{P}=\left(\frac{1}{2} \Delta E_{v w}^{P}\right)^{2} \sqrt{\frac{\pi^{3}}{E_{r} k_{B} T \hbar^{2} \omega_{e f f}^{2}}} \text { when } \quad \kappa_{v w}^{P} \quad<1 \\
& \Delta \varepsilon_{v w}^{P}=\frac{1}{\pi} \hbar \Omega_{P} \exp \left\{-\frac{1}{\hbar} \int_{l e f t}^{r i g h t}\left\{2 m_{P}\left[U_{v w}\left(r_{P}\right)-\varepsilon_{v w}^{P}\right]^{\frac{1}{2}}\right\}\right\}
\end{aligned}
$$

where $m_{P}$ is the proton mass. The integration limits represent the proton tunneling barrier. We note: (1) The same quadratic free energy relation, eq.(3) as for ET is recognized.

(2) Statistical averaging over reactants', $\varepsilon_{R v}^{P}$, and summation over products' proton vibrational states, $\varepsilon_{P_{w}}^{P}$ are included. This is important for KIEs and as the inverted free energy range is approached but otherwise often reduces to dominance of the ground vibrational states $v=w=0$.

(3) The transmission coefficient, $\kappa_{v w}^{P}$, is determined by the energy splitting of the proton vibrational levels at resonance, $\Delta \varepsilon_{v w}^{P} . \Delta \varepsilon_{v w}^{P}$ is in turn determined by the proton vibrational frequency, $\Omega_{P}$, and the proton tunneling barrier, $U_{v w}\left(r_{P}\right)$ along the proton coordinate, $r_{P}$, at the nonequilibrium (fluctuational) environmental resonance configuration, eq.(5).

The character of the PT reaction changes entirely in the totally adiabatic limit of strong proton donor-acceptor interaction, where the opposite inequality of eq.(4) applies. $\kappa_{v w}^{P} \rightarrow 1$ in this limit where tunneling features appear differently. The rate constant, eq.(2) reduces to

$$
W_{R P}^{v w}=\frac{\omega_{e f f}}{2 \pi} \exp \left(-\frac{G_{v w}^{\neq}}{k_{B} T}\right)
$$

Tunneling remains by the quantum mechanical indices $\mathrm{v}$ and $\mathrm{w}$ and the modified $G_{v w}^{\ddagger}$-form

$$
G_{v w}^{\neq} \rightarrow G_{v w}^{ \pm}-[\alpha(1-\alpha)]^{\frac{1}{2}} \Delta \varepsilon_{v w}^{P} \approx G_{v w}^{\neq}-\frac{1}{2} \Delta \varepsilon_{v w}^{P}
$$

where $\alpha$ is the Brønsted coefficient, cf. below. The proton tunneling features are thus now reflected by a lower activation free energy rather than in the explicit appearance of a pre-exponential 


\section{Free Energy Relations and KIEs}

Free energy relations and the KIEs are established outcomes of the formalism summarized in eqs.(1)-(8). Extensions to novel ranges and systems are discussed in Sections 4 and 5.

\subsection{The Brønsted relation}

When only the vibrational ground states are important, the Brønsted coefficient is

$$
\alpha_{00} \approx-k_{B} T d \ln W_{R P}^{00} / d \Delta G^{0} \approx \frac{1}{2}+\frac{\Delta G^{0}}{2 E_{r}}
$$


The Brønsted coefficient $\alpha$ varies between zero and unity as $\Delta G^{0}$ varies from $-E_{r}$ to $E_{r}$. $\alpha$ is $1 / 2$ for thermoneutral processes, $\Delta G^{0}=0$. The following features modify this simple view:

- Excited high-frequency vibrational proton/H-atom stretching modes $\left(3000 \mathrm{~cm}^{-1}\right.$ vs. $k_{B} T \approx 200$ $\mathrm{cm}^{-1}$ ) are in fact important even for PT processes in the "normal" free energy range, $\left|\Delta G^{0}\right|<E_{r}$. Solvent librational $\left(800 \mathrm{~cm}^{-1}\right)$ or proton bending modes $\left(\approx 1500 \mathrm{~cm}^{-1}\right)$ are other tunneling modes. A more general form of eq.(9) is therefore

$\alpha \approx-k_{B} T d \ln W_{R P} / d \Delta G^{0} \approx \frac{1}{2}+\frac{\left(\Delta G^{0}+w^{*} \hbar \Omega_{p}-v^{*} \hbar \Omega_{p}\right)}{2 E_{r}}$

Where $v^{*}$ and $w^{*}$ are the values of $v$ and $w$ that give the maximum contribution to the rate constant at given $\Delta G^{0} \cdot v^{*} \approx w^{*}=0$ as long as $\left|\Delta G^{0}\right|<<E_{r}$ but finite $w^{*}\left(v^{*}\right)$ gains importance as $\left|\Delta G^{0}\right|$ approaches $E_{r}$ and extends the $\Delta G^{0}$-range over which $\alpha$ changes from zero to unity.

- Chemical bond deformation in PT/H-atom transfer processes is much stronger than in most ET processes. Anharmonic potentials such as the Morse and Rosen-Morse potentials ${ }^{5,17,18}$ are therefore needed in more precise data fitting. These potentials give smaller vibrational level spacings, eqs.(1)(5), attenuated tunneling, and broader approximately linear ranges of the free energy relations.

- The Brønsted coefficient for PT between strongly H-bonded O- and N-acids and -bases often varies between zero and unity within a few $p K_{a}$-units. This range is much wider for $\mathrm{H}$-bonded $\mathrm{C}$ acids and -bases. Such patterns accord with major pre-organization (work terms) in the former class, while the reorganization free energy in the PT step itself is small. Pre-organization is less important for $\mathrm{C}$-acid/bases in which, however, significant low-frequency intramolecular reorganization increases $E_{r}$, extending the approximately linear free energy range.

- Free energy relations of photochemically induced PT reactions have been extended from the "normal" $\left(\left|\Delta G^{0}\right|<E_{r}\right)$, via the activationless $\left(\left|\Delta G^{0}\right| \approx E_{r}\right)$ to the "inverted" free energy region $\left(\left|\Delta G^{0}\right|>E_{r}\right.$ ). Dating back to early "energy gap" relations for solid state electronic relaxation ${ }^{46}$, this notion is also long well understood for ET processes ${ }^{4,5,16}$.

Fig.3 


\subsection{Kinetic Deuterium Isotope effects}

$\mathrm{PT} / \mathrm{H}$-atom transfer processes are unique cases for direct observation of room temperature nuclear tunneling in the form of the kinetic deuterium and tritium isotope effects. The KIE offers an experimental approach to subtle details of the $\mathrm{PT} / \mathrm{H}$-atom dynamics such as $\mathrm{PT} / \mathrm{H}$-atom transfer distance, nature of the harmonic or anharmonic PT/H-atom double-well potential, and the role of $\mathrm{PT} / \mathrm{H}$-atom gating. The physical origin of the KIE is different in partially and totally adiabatic processes. The dominating effect in the former class is tunneling which is more facile for $\mathrm{PT} / \mathrm{H}-$ 
atom than for DT/D-atom transfer. The KIE in the totally adiabatic limit is instead dominated by different splitting of the potential (free) energy surfaces, eq.(8).

\subsubsection{The KIE in Partially Adiabatic PT/H-atom Transfer Processes}

\subsubsection{The Role of Gating}

PT/H-atom transfer between structural equilibrium sites would involve PT/H-atom transfer distances in the range $\Delta r_{H}^{0}=0.6-1.0 \AA$. Such a distance is prohibitive for fast PT/H-atom tunnelling and gives unphysically large values of the $\mathrm{KIE}^{4,5,17,18}$. Gating, i.e. expenditure of activation energy in bringing the donor and acceptor group closer to achieve a much smaller PT/H-atom transfer distance is therefore crucial and much more important than for ET. Due to the large D/H mass ratio, the gating feature is more conspicuous for DT than for PT, i.e. the DT/D-atom transfer distance is usually smaller and requires stronger thermally activated gating than for PT/H-atom transfer. As reported early ${ }^{4,8}$, much of the apparent activation energy of the KIE is associated with this effect, cf. also later work ${ }^{11,12,35,45}$.

The PT/H-atom transfer rate constant can be given the approximate form, eqs.(1)-(5)

$k_{H} \approx \Phi\left(R_{H}^{*}\right) W_{R P}^{00}\left(r_{H}^{*} ; R_{H}^{*}\right) \Delta R_{H}$

when $\left|\Delta G^{0}\right|<E_{r} . \Phi\left(R_{H}\right)$ is the probability that mutual approach takes the donor and acceptor units along the translational coordinate $R_{H}$ up to the value $R_{H}^{*}$ where PT/H-atom transfer over the distance $\Delta r_{H}^{*}<\Delta r_{H}^{0}$ occurs. $\Delta R_{H}$ is the range of $R_{H}$ over which PT/H-atom transfer is feasible.

All the terms in eq.(11) are sensitive to isotope substitution. $\Phi\left(R_{H}\right)$ usually decreases with increasing $R_{H}$ whereas $W_{R P}^{00}\left(r_{H}^{*} ; R_{H}^{*}\right)$ increases rapidly due to the increasing overlap of the proton wave functions or tunneling splittings. The KIE for the $0 \rightarrow 0$ transition is therefore, eqs. (1)-(5)

$$
K I E \approx \frac{\omega_{e f f}^{H}}{\omega_{e f f}^{D}} \frac{\Phi\left(R_{H}^{*}\right)}{\Phi\left(R_{D}^{*}\right)} \frac{\kappa_{R P}^{00, H}\left(\Delta r_{H}^{*} ; R_{H}^{*}\right)}{\kappa_{R P}^{00, D}\left(\Delta r_{D}^{*} ; R_{D}^{*}\right)} \rightarrow \frac{\omega_{e f f}^{H}}{\omega_{e f f}^{D}} \exp \left[\frac{m_{p}^{H} \Omega_{P}}{2 \hbar} \sqrt{2}\left(\Delta r^{*}\right)^{2}\right]
$$

where $\kappa_{R P}^{00, H}$ and $\kappa_{R P}^{00, D}$ are given by eqs.(4) and (5) for $v=w=0$. The second term applies when $\Delta r_{H}^{*}=\Delta r_{D}^{*}=\Delta r^{*}$. As noted, in general $\Delta r_{H}^{*}>\Delta r_{D}^{*}$ but $\kappa_{R P}^{00, H}\left(\Delta r_{H}^{*}\right)$ still significantly exceeds 


\subsubsection{The KIE in the Totally Adiabatic Limit}

The origin of the KIE in this limit is still rooted in tunneling but takes a different form ${ }^{5,12,15}$

$$
K I E \approx \frac{\omega_{e f f}^{H}}{\omega_{e f f}^{D}} \frac{\Phi\left(R_{H}^{*}\right)}{\Phi\left(R_{D}^{*}\right)} \exp \left\{\frac{\delta \Delta \varepsilon_{00}^{H, D}[\alpha(1-\alpha)]^{\frac{1}{2}}}{k_{B} T}\right\} ; \quad \delta \Delta \varepsilon_{00}^{H, D}=\Delta \varepsilon_{00}^{H}-\Delta \varepsilon_{00}^{D}
$$

Insertion of the symmetry factor $\alpha$ from eq.(9) recasts eq.(13) in a volcano type form, i.e. 


$$
K I E \approx \frac{\omega_{e f f}^{H}}{\omega_{e f f}^{D}} \frac{\Phi\left(R_{H}^{*}\right)}{\Phi\left(R_{D}^{*}\right)} \exp \left\{\frac{\delta \Delta \varepsilon_{00}^{H, D} \frac{1}{2}}{2 k_{B} T}\left[1-\left(\frac{\Delta G^{0}}{E_{r}}\right)^{2}\right]^{\frac{1}{2}}\right\}
$$

with a maximum at $\Delta G^{0} \approx 0$ as for the partially adiabatic limit.

Eqs.(13) and (14) offer two observations. One is that the KIE is associated solely with the activation free energy and therefore closer in keeping with classical KIE views as vibrational zero point activation energy differences in the reactants' and transition states. The other observation is that the KIE decreases with increasing transfer distance. This "inverted" distance dependence is caused by the decreasing $\delta \Delta \varepsilon_{00}^{H, D}\left(\delta \Delta \varepsilon_{v w}^{H, D}\right)$ as the distance increases. As this feature only applies in the totally adiabatic limit, the overall distance dependence of the KIE passes a minimum when the proton/Hatom transfer distance crosses over from the totally adiabatic limit at small distances and the partially adiabatic limit at larger distances.

\section{Some systems}

The conceptual PT/H-atom transfer framework above has been brought to accord in considerable detail with a wide variety of processes. These include classical $\mathrm{O}-, \mathrm{N}-$, and $\mathrm{C}$-based acid base catalysis $^{4,5,17,18}$, low-temperature H/D-atom transfer processes ${ }^{29}$ where nuclear tunneling in the gating mode is a conspicuous feature ${ }^{4,5}$, and enzyme radical processes with large and temperature dependent KIE's with temperature coefficients in the $\mathrm{kcal}^{\mathrm{mole}} \mathrm{e}^{-1}$ range $^{9,13,14,45}$. We consider here some other applications of PT/H-atom transfer theory.

\subsection{PT in strongly H-bonded Environments}

This notion is illustrated by the excess proton conductivity (EPC) in aqueous solution, Fig. $6^{26,31,32,48-51}$ but carries over to environments that are, in such a context novel. These include proton conductors or proton pumping in membrane-spanning protein complexes ${ }^{19-22}$, or pore confined EPC in fuel cell membranes such as Nafion ${ }^{\circledR 26,52,53}$. The dominating room temperature molecular species is believed to be the Zundel ion, $\mathrm{H}_{5} \mathrm{O}_{2}{ }^{+}$with the proton located symmetrically between two strongly solvating but highly labile water molecules. This suggests that the excess proton is substantially "delocalized" and that bulk EPC is a case for coupled two- (or multiple) $\mathrm{PT}^{32-34}$. EPC is induced by subtle dynamic interplay between the hydrogen bonding water molecules. Only tiny structural shifts are needed to effect efficient proton translocation where the 
activation free energy is entirely in the gating mode(s), with virtually no PT barrier left. Such views may carry over to PT along flexible amino or carboxylate groups lining biological proton transport channels, or the pore lining sulfonate groups in Nafion ${ }^{\circledR}$ fuel cell membranes.

EPC (the Grotthuss mechanism) has long been a target ${ }^{54,55}$ and is also presently in focus of largescale computations ${ }^{30,31,48-51}$. EPC can be accommodated within the analytical theoretical frames above subject to some observations. The efficiency of EPC is rooted in the strong H-bonds in the proton transmitting molecular entities $\left(\mathrm{H}_{5} \mathrm{O}_{2}{ }^{+}\right)$and in the solvation by the closest water molecules. H-bonding and interaction between the proton donor and acceptor molecules, Fig. 6 can further be so strong that the PT barrier disappears altogether and the proton is accommodated in a singlerather than a double-well potential. Observable PT dynamics including the KIE is therefore dominated by the solvent dynamics, in keeping with observed KIEs close to $\sqrt{2}$, eqs.(13) and (14). PT in strongly H-bonded systems represented by the scheme in Fig.5 is monitored by the diffusion coefficient, $D_{n}$ or ionic mobility, $\mu_{n}$ related to the (totally adiabatic) PT rate constant, $W_{n}$ by

$$
\begin{array}{ll}
D_{n}=P_{n} W_{n} a_{n}^{2} ; & W_{n}=N_{n} \frac{\omega_{e f f}}{2 \pi} \exp \left(-\frac{G_{n}^{\mp}}{k_{B} T}\right) \\
\mu_{n}=\frac{e D_{n}}{k_{B} T} ; & \mu_{o b s}=\frac{e}{k_{B} T} \sum_{n} P_{n} D_{n} a_{n}^{2}
\end{array}
$$

The proton conducting species $\left(n\right.$, say $\mathrm{H}_{3} \mathrm{O}^{+}, \mathrm{H}_{5} \mathrm{O}_{2}{ }^{+}, \mathrm{H}_{9} \mathrm{O}_{4}{ }^{+}$) are represented by the distribution function, $P_{n}$. $G_{n}^{\neq}$is the activation free energy of species $n$, and $a_{n}$ the proton jump distance specific to the PT species and elementary PT step (single- or double-PT, Zundel or Eigen ion etc. ${ }^{34}$ ). $N_{n}$ is a structural factor which depends on the number of PT directions of species $n$, and $e$ the electronic charge. This approach resembles classical views on EPC over wide temperature ranges ${ }^{55}$ but with the PT processes of different proton complexes now brought within condensed matter PT theory. As the strongly adiabatic limit prevails focus in analytical approaches to eq.(15) is on the activation free energy. Distinction between gated PT in double-well and single-well potentials is convenient.

\subsubsection{PT and Proton Conductivity in Double-well Potentials}

Fig.5 
Single- and double-PT steps can be envisaged. Consideration of PT in "adjacent" and "remote" Zundel complexes identifies the following gating and bulk nuclear modes ${ }^{15,34,38}$ :

- The distance between the proton donor, $\mathrm{H}_{5} \mathrm{O}_{2}{ }^{+}$, and acceptor $\mathrm{H}_{2} \mathrm{O}$ molecular centres.

- The distance between the proton donor, $\mathrm{H}_{5} \mathrm{O}_{2}{ }^{+}$and a (set of) local solvent molecule(s), $\mathrm{S}_{\mathrm{D}}$.

- The distance between the proton acceptor, $\mathrm{H}_{2} \mathrm{O}$ and a second set of local solvent molecules, $\mathrm{S}_{\mathrm{A}}$.

- Collective solvent coordinates representing the inertial polarization outside the reaction centre. These notions and the prevalence of the $\mathrm{H}_{5} \mathrm{O}_{2}{ }^{+}$ion implies that EPC is a case for "long-range" PT in the sense that more than a single proton is involved in the elementary PT steps. The views can be incorporated in representations of the different terms in eq.(15), say PT involving $\mathrm{H}_{3} \mathrm{O}^{+}$, adjacent and remote Zundel ions etc. ${ }^{15,34,38}$. As an example, using Morse potentials for the local mode dynamics, the activation free energy for the adjacent Zundel ion-based mechanism takes a simple form $^{15,38}$

$$
G^{ \pm}=\frac{1}{4} E_{r}+\frac{1}{2} D_{\text {Zundel }}-\frac{\Delta \varepsilon_{00}^{\text {Zundel }}}{8\left(1-\frac{\Delta \varepsilon_{00}^{\text {Zundel }}}{4 D_{\text {Zundel }}}\right)}
$$

where $D_{\text {Zundel }}$ is the dissociation energy of the Zundel complex and $\Delta \varepsilon_{00}^{\text {Zundel }}$ the isotope dependent resonance splitting of the two-PT in the adjacent Zundel complex mechanism.

\subsubsection{PT and Proton Conductivity in Single-well Potentials}

The proton potential in complexes with strong $\mathrm{H}$-bonds in both the initial and final states can reduce to a single-well potential with the proton shifted closer to either the donor or the acceptor ${ }^{15,38 b}$. There are indications from quantum path integral and other computational approaches ${ }^{32,52-55}$ that this limit could apply to the EPC mechanism in aqueous solution.

Fig.6

Fig.6 shows the PT sequence. The proton is located initially in a single-well potential with a minimum closest to the proton donor, due to the interaction between the PT complex and (a) local water molecule(s), $S_{D}$. Configurational fluctuations induce a shift of $S_{D}$ with both deformation and a 
$U(s)=V_{S C}(s)+E_{p}(s) ; \quad G^{\neq}=V_{S C}\left(s^{*}\right)-V_{S C}\left(s_{D 0}\right)+E_{p}\left(s^{*}\right)-E_{p}\left(s_{D 0}\right)$

where $V_{S C}(s)$ is the interaction energy and $E_{p}(s)$ the ground state proton vibrational energy. $s^{*}$ and $s_{D O}$ are the values of $s$ in the transition state and the initial equilibrium state, respectively.

Eqs.(17) and (18) can be combined with harmonic, Morse or other potentials. A key difference from PT in double-well potentials is that the activation free energy is determined by the interaction energy and the proton vibrational zero point energy differences between the transition and initial states. This observation carries over to the KIE

$$
K I E \approx \frac{\omega_{e f f}^{H}}{\omega_{e f f}^{D}} \exp \left\{-\frac{\left[E_{H}\left(s^{*}\right)-E_{D}\left(s^{*}\right)\right]}{k_{B} T}\right\}
$$

since the interaction energies $V_{S C}(s)$ are the same for both isotopes. Eq.(19) can give KIEs either larger or smaller than unity depending on whether the single-well PT potential in the transition state is shallower or steeper than in the initial equilibrium state.

\subsection{PT in the Inverted Free Energy Free Energy Region}

Savéant and associates ${ }^{39}$, and Peters and associates ${ }^{40,41}$ have opened the area of PT processes in the inverted free energy region. The studies of Savéant and associates addressed PT in a series of diphenylmethane/diphenylmethyl anion base systems. Those of Peters and associates focused on photo-induced PT between a series of benzophenones and N,N'-dimethylaniline or N,N'-dimethylp-toluinide. A maximum in the Brønsted relation was observed in either study. The fall-off of the rate constant on the strongly exergonic side was significant in the former case, i.e. between one and two orders of magnitude over a free energy range of about $0.4 \mathrm{eV}$, but only a factor of two or less over $0.2-0.3 \mathrm{eV}$ in the latter. The inverted driving force range was about the same as the vibrational energy quantum of the proton stretching mode $(0.3 \mathrm{eV})$ in either case. 
As an illustration, Fig.7 shows two free energy plots based on eqs.(1)-(5) and displaced harmonic potential surfaces extending into the inverted region. The low-frequency reorganization free energy was taken as $0.9 \mathrm{eV}$ and the proton vibrational frequency $3000 \mathrm{~cm}^{-1}$ corresponding to a vibrational energy of $\hbar \Omega_{H} \approx 0.3 \mathrm{eV}$. The proton coupling constant $\Delta_{H}=\frac{1}{2}\left(m \Omega_{H} / \hbar\right)\left(\Delta r_{H}^{*}\right)^{2}$ was 5 (dashed line) and 2 (fully drawn line), or $\Delta r_{H}^{*} \approx 0.3$ and $0.2 \AA$, respectively. The following is noted:

- Several excited vibrational states in the products' electronic state contribute around the activationless and in the inverted free energy regions, dominated by $w^{*} \approx-E_{r}-\Delta G^{0}\left(-\Delta G^{0}>E_{r}\right)$.

- $\Delta_{H}=5$ corresponds to values that accord with many cases of large KIE $(\geq 10$; for approximately thermoneutral PT processes!) based on displaced harmonic potential surfaces. The rate constants, however, only fall off at large $\left|\Delta G^{0}\right|$. The apparent discrepancy between the data ${ }^{39-41}$ and the expectation that excited proton vibrational states would cause the rate to stay in the activationless region rather than move into the inverted region was denoted as a "conundrum" 41 . In contrast $\Delta_{H}=$ 2 gives an almost symmetric maximum at $\Delta G^{0}=-1.2 \mathrm{eV}$ corresponding to a shift of about one vibrational quantum from $\Delta G^{0}=-0.9 \mathrm{eV}$ when no high-frequency modes are present. This maximum appears at lower $\left|\Delta G^{0}\right|$ if the low-frequency reorganization free energy is smaller.

- The "conundrum" 41 must, however, be viewed along with the very shallow observed maximum and small KIE (1.1-2). The latter might appear to require a very small PT distance or coupling constant but the KIE falls off strongly as the activationless free energy region is approached or traversed due to the increasing importance of excited proton vibrational states ${ }^{17,18}$. The maximum is more pronounced in the data by Savéant and associates ${ }^{39}$ but no KIE data were reported here. Diffusion effects were not addressed.

- Replacing the harmonic potentials with Morse or other shallower potentials would lead to a more pronounced maximum. The small KIE and PT distances, however, still require attention and perhaps remain as the "real" conundrum, if there is one.

Figure 7

\subsection{Two Cases of Unusual KIE Behaviour}

The KIE sometimes disclose behaviour unexpected from views such as those discussed in Sections 2 and 3 . We consider two such cases. 


\subsubsection{Attenuation of Intrinsic KIE by Solvent Relaxation}

Hydrolytic enzyme systems often display KIEs barely in excess of $\sqrt{2}$. Although small by the formalism summarized in Sections 2 and 3, the observed small values may conceal significantly larger intrinsic values which are, however, attenuated by other rate controlling factors such as solvent relaxation in particular.

Carboxypeptidase A catalysis of small peptide and ester hydrolysis offers such a case. The observed comparative KIE of ester (Bz-Gly-O-Ph-Lac) and peptide (Bz-Gly-Phe) hydrolysis are 1.7 and 1.3, respectively. However, the rate constant of the former but not of the latter displays strong (subexponential) solvent viscosity dependence ${ }^{42}$. By stochastic chemical rate theory as applied to such kinds of systems, a useful operational (adiabatic) rate constant form is ${ }^{56}$

$$
W_{R P}^{c a t}=\left(W_{R P}^{-1}+W_{R P, \text { friction }}^{-1}\right)^{-1}
$$

$$
W_{R P, \text { friction }} \approx \tau_{\text {rel }}^{-\delta}\left(\frac{\omega_{e f f}}{2 \pi}\right)^{1-\delta} \exp \left(-\frac{\gamma G^{\ddagger}}{k_{B} T}\right)
$$

$W_{R P}$ is given by suitable choice among eqs.(6) and (7), $\gamma=E_{s} / E_{r}$, cf. eqs.(3) and (10) where $E_{s}$ is the (vibrationally) damped solvent part of $E_{r}$ and $\delta$ is a constant that represents the response of the protein dynamics to the solvent relaxation of which $\tau_{\text {rel }}$ is the relaxation time. Eq.(20) that applies to ester hydrolysis shows that the KIE can indeed attenuate the KIE, giving an intrinsic value of 2.22.7, significantly larger than the observed value. The KIE and viscosity based data also point to different mechanisms for ester and peptide hydrolysis.

\subsubsection{Unusual KIEs in Protein Electron Transfer Processes}

Intramolecular electron transfer (ET) between a reduced disulfide group and the copper centre in the blue redox metalloprotein Pseudomonas aeruginosa azurin has been reported to display KIE values less than unity (0.6-0.8) strongly reflected in both the rate constants and the apparent activation parameters (temperature coefficients) ${ }^{43}$. Such values exclude proton/deuteron tunneling as the primary cause of the KIE. 
Based on analysis of the temperature variation of both the driving force and the rate constants in $\mathrm{H}_{2} \mathrm{O}$ and $\mathrm{D}_{2} \mathrm{O}$, the conclusion was reached that differences in both thermodynamic (redox potential, solvation) and physical properties (thermal expansion) of the solute protein system in $\mathrm{H}_{2} \mathrm{O}$ and $\mathrm{D}_{2} \mathrm{O}$ are large enough to invoke observable KIE which can, furthermore assume such unusual values. KIE values in composite protein or other macromolecular systems that are not significantly in excess of unity can, therefore conceal a composite range of other features than proton tunneling.

\section{New Approaches - towards PT/H-atom Transfer at the Single-molecule Level}

\subsection{Single-molecule Electrochemical Electron Transfer}

The introduction of scanning tunneling microscopy (STM) has opened novel perspectives in molecular charge transfer science. Both molecular structural and electronic tunneling properties of single molecules can be addressed. Extension of this technology, with supporting theoretical frames to electrochemically controlled in situ STM offers other perspectives for single-molecule mapping directly in aqueous electrolyte ${ }^{57-59}$. Electrochemically controlled in situ STM of single-molecule ET dynamics has offered novel understanding of the fundamental ET process of both non-redox and redox molecules, mapping of tunneling and stochastic properties, and even disclosed new ET phenomena $^{57,58}$. A strategy where single-molecule environments can be extended to singlemolecule PT/H-atom transfer has recently been offered ${ }^{44}$. No data are available presently, but once achieved, single-molecule PT/H-atom transfer perspectives such as experimentally elusive systematic distance dependence of PT/-atom transfer rate and KIE could be addressed.

\subsection{Single-molecule Proton/Hydrogen Atom Transfer}

As noted, recent efforts have opened new approaches to interfacial molecular electron transfer (ET) at levels of resolution of the single molecule ${ }^{57}$. Local environments have been both electrochemical and non-electrochemical pure and modified metal surfaces ${ }^{57,59}$, particularly Au(111)- and Pt(111)surfaces. Three-electrode systems have been the basis for tunneling spectroscopy, and new concepts and formalism explored with notable experimental support ${ }^{57}$.

Field-induced PT reactions with focus on asymmetric double-PT were suggested early as a basis for single-molecule information storage elements ${ }^{60}$. Double-PT processes have been in later experimental $^{61}$ and theoretical focus ${ }^{62}$ but not in the context of single-molecule function and control. No data for single-molecule PT/H-atom transfer that match the present level of singlemolecule ET are presently available. Unlike ET processes, PT and PCET kinetics studies have thus 
so far been restricted to ensemble averages. As for ET, this conceals details of the microscopic mechanism. With a view on the notable recent progress in single-molecule interfacial ET and on the long-term efforts in the chemical physics of PT/H-atom transfer in chemistry and biology, we have recently introduced a theoretically framed scheme for single-molecule PCET processes close to a level that can, expectably be addressed experimentally.

\subsubsection{A scheme for Single-molecule Concerted PCET}

The scheme is based on electrochemical scanning tunneling microscopy (in situ STM). As for single-molecule ET, two types of transitions can be distinguished, i.e. concerted PCET and stepwise $\mathrm{ET} / \mathrm{PT}^{15,63}$ of which we address here the former. The limit is $\mathrm{H}$-atom transfer but with interfacial electrochemical ET between the molecule and the enclosing electrodes controlling the overall process. In this sense $\mathrm{H}$-atom transfer does not differ formally from $\mathrm{PT}^{4-8,63}$.

The PCET scheme is shown in Fig. 8. A molecule, $\boldsymbol{A}$ (proton or H-atom acceptor) is attached to a metal electrode $M_{L}$ (STM substrate). The second metal electrode $M_{R}$ is the tip (subscripts denote left and right electrodes) with the proton or H-atom donor, $\boldsymbol{D}$ attached. The proton may be bound to either of the molecules, forming the molecular states $\mathrm{AH}$ and $\mathrm{DH}$. The tunneling contact is immersed in electrolyte/buffer solution. The bias voltage $e V$ is defined as in Fig.8A.

Fig. 8

Two PCET processes can take place in the complex $\mathrm{M}_{\mathrm{L}}-\mathrm{A}-\mathrm{H}-\mathrm{D}-\mathrm{M}_{\mathrm{R}}$

$\mathrm{M}_{\mathrm{L}}(\mathrm{e})-\mathrm{A}+\mathrm{H}-\mathrm{D}-\mathrm{M}_{\mathrm{R}} \leftrightarrow \mathrm{M}_{\mathrm{L}}-\mathrm{A}-\mathrm{H}+\mathrm{D}-\mathrm{M}_{\mathrm{R}}(\mathrm{e})$

$\mathrm{M}_{\mathrm{L}}-\mathrm{A}-\mathrm{H}+\mathrm{D}-\mathrm{M}_{\mathrm{R}} \leftrightarrow \mathrm{M}_{\mathrm{L}}-\mathrm{A}+\mathrm{H}-\mathrm{D}-\mathrm{M}_{\mathrm{R}}$

Reaction (21) is the electrochemical process that involves synchronous transfer of the proton between the molecules $\boldsymbol{A}$ and $\boldsymbol{D}$ and two electrons between the left electrode and $\boldsymbol{A}$, and the right electrode and $\boldsymbol{D}$. The overall process is thus concerted proton-coupled two-ET, PC-2ET. The second process is purely chemical $\mathrm{H}$-atom transfer between the molecules. Due to the strong electrostatic potential created by proton, the highest occupied electron valence level and the lowest unoccupied level of the HD molecule are located well below and well above the Fermi level, respectively of the 
right electrode so that pure two-step ET is suppressed. The rate of reaction (22), $k_{\mathrm{H}}^{\mathrm{AD}}$ is independent of the electrochemical potentials and should be exothermic (i.e. $k_{\mathrm{H}}^{\mathrm{DA}}<<k_{\mathrm{H}}^{\mathrm{AD}}$ ) for the system to operate at positive $V$. The rate of the forward reaction (21) $k_{\mathrm{p}}^{\mathrm{DA}}$ increases with increasing bias voltage whereas the reverse reaction rate, $k_{\mathrm{p}}^{\mathrm{AD}}$ decreases. PT thus dominates $\boldsymbol{D} \rightarrow \boldsymbol{A}$ hydrogen transfer and $\mathrm{H}$-atom transfer the reverse transition.

The operational mode at positive bias voltage is that the first step is PT from $\boldsymbol{D}$ to $\boldsymbol{A}$ with simultaneous transfer of two electrons (from $\boldsymbol{D}$ to the right electrode and from the left electrode to $\boldsymbol{A})$. The second step that closes the cycle is H-atom transfer from $\boldsymbol{A}$ to $\boldsymbol{D}$. A single electron thus passes the electrochemical contact in each cycle. Ideally the system allows studying the potential dependence of the single-molecule interfacial rate constants and the distance dependence of the $\mathrm{H}$ atom transfer rate and the KIEs, by varying the tip-substrate distance.

\subsubsection{Electric Current and Single-molecule Rate Constants for Concerted ET/PT}

The single-molecule steady-state electric current through the contact shown in Fig. 8 is

$$
j_{\text {conct. }}=e\left(P_{\mathrm{DH}} k_{\mathrm{p}}^{\mathrm{DA}}-P_{\mathrm{AH}} k_{\mathrm{p}}^{\mathrm{AD}}\right)
$$

where $P_{\mathrm{DH}}$ and $P_{\mathrm{AH}}$ are the probabilities to find the proton in molecule $\boldsymbol{D}$ and $\boldsymbol{A}$, respectively. The superscripts denote the direction of PT ( $\boldsymbol{D}$ to $\boldsymbol{A}$, DA, or $\boldsymbol{A}$ to $\boldsymbol{D}, \mathrm{AD})$. Eq.(23) can be recast as

$$
j_{\text {conct. }}=e \frac{k_{\mathrm{p}}^{\mathrm{DA}} k_{\mathrm{H}}^{\mathrm{AD}}-k_{\mathrm{p}}^{\mathrm{AD}} k_{\mathrm{H}}^{\mathrm{DA}}}{k_{\mathrm{p}}^{\mathrm{DA}}+k_{\mathrm{H}}^{\mathrm{AD}}+k_{\mathrm{p}}^{\mathrm{AD}}+k_{\mathrm{H}}^{\mathrm{DA}}}
$$

where $k_{\mathrm{H}}$ with appropriate superscripts are the single-molecule forward and reverse rate constants for $\mathrm{H}$-atom transfer between $\mathrm{A}$ and $\mathrm{D}$, related by

$$
k_{\mathrm{H}}^{\mathrm{DA}}=k_{\mathrm{H}}^{\mathrm{AD}} \exp \left(\frac{G_{D H}^{0}-G_{A H}^{0}}{k_{\mathrm{B}} T}\right) ; \quad k_{\mathrm{p}}^{\mathrm{AD}}=k_{\mathrm{p}}^{\mathrm{DA}} \exp \left(\frac{G_{A H}^{0}+\varepsilon_{\mathrm{FR}}-G_{D H}^{0}-\varepsilon_{\mathrm{FL}}}{k_{\mathrm{B}} T}\right)
$$


with $K_{\mathrm{DA}}(<<1)$ being the equilibrium constant.

Eq.(26) shows that if, at small $V k_{\mathrm{p}}^{\mathrm{DA}}$ is small compared to $k_{\mathrm{H}}^{\mathrm{AD}}$ the dependence of the current on the potential is determined by the former.

$$
j_{\text {conct. }} \approx e k_{\mathrm{p}}^{\mathrm{DA}}
$$

while at large $V$ when $k_{\mathrm{p}}^{\mathrm{DA}} \gg k_{\mathrm{H}}^{\mathrm{AD}}$ the current is determined by the latter

$$
j_{\text {conct. }} \approx e k_{\mathrm{H}}^{\mathrm{AD}}
$$

The detailed expressions for the single-molecule rate constants depend on the microscopic mechanism of the PT or H-atom transfer ${ }^{4-8,17,18}$. Restricting ourselves to the partially adiabatic limit in both interfacial electrochemical ET and PCET between the molecules, the H-atom transfer rate constant, eq.(22) can be represented in the form, cf. eqs.(1)-(5)

$$
k_{\mathrm{H}}^{\mathrm{AD}}=\frac{\omega_{e f f}}{2 \pi} Z_{\mathrm{H}}^{-1} \sum_{m, n} \kappa_{v w}^{\mathrm{H}} e^{-\frac{\varepsilon_{R v}-\varepsilon_{R 0}}{k_{\mathrm{B}} T}} \exp \left(-G_{v w}^{\neq} / k_{\mathrm{B}} T\right)
$$

where $\kappa_{v w}^{\mathrm{H}}$ and $G_{v w}^{\neq}$are the transmission coefficient and activation Gibbs free energy for H-atom transfer between the initial and final $\mathrm{H}$-atom vibrational states $v$ and $w, Z_{\mathrm{H}}$ the vibrational partition function of these states in the initial electronic state and $\varepsilon_{R v}$ the energy of the $v$ 'th $\mathrm{H}$-atom vibrational state in the reactants' state. 
The concerted ET/PT is more complicated

$$
k_{\mathrm{p}}^{\mathrm{DA}}=\int d \varepsilon_{\mathrm{L}} d \varepsilon_{\mathrm{R}} f_{\mathrm{L}}\left(\varepsilon_{\mathrm{L}}\right)\left[1-f_{\mathrm{L}}\left(\varepsilon_{\mathrm{R}}\right)\right] \rho_{\mathrm{L}}\left(\varepsilon_{\mathrm{L}}\right) \rho_{\mathrm{R}}\left(\varepsilon_{\mathrm{R}}\right) W_{\mathrm{H}^{+}}\left(\varepsilon_{\mathrm{L}}, \varepsilon_{\mathrm{R}}\right)
$$

where $f_{\mathrm{M}}$ and $\rho_{\mathrm{M}}(\mathrm{M}=\mathrm{L}, \mathrm{R})$ are the Fermi functions and densities of electronic states in the electrodes at the electronic energy level $\varepsilon_{\mathrm{M}} . W_{H^{+}}$is the transition probability per unit time between a given pair of electronic energy levels $\left(\varepsilon_{L}, \varepsilon_{R}\right)$ and has a form similar to eq.(1)

$$
W_{\mathrm{H}^{+}}\left(\varepsilon_{L}, \varepsilon_{R}\right)=\frac{\omega_{e f f}}{2 \pi} Z_{\mathrm{H}^{+}}^{-1} \sum_{m, n} \kappa_{v w}^{\mathrm{H}^{+}} e^{-\frac{\varepsilon_{R v}-\varepsilon_{R 0}}{k_{\mathrm{B}} T}} \exp \left(-\frac{G_{v w}^{\neq, H^{+}}\left(\varepsilon_{L}, \varepsilon_{R}\right)}{k_{B} T}\right)
$$

All the quantities have the same meaning as in eq.(29) but now refer to PC-2ET.

The transmission coefficients for both steps take the same form as in eq.(4). The activation Gibbs free energy depends on the electric potential ${ }^{64}$

$$
\begin{gathered}
G_{v, w}^{\neq \mathrm{H}^{+}}=\left[E_{r}-\Delta G^{0}+\varepsilon_{\mathrm{L}}-\varepsilon_{\mathrm{FL}}-\varepsilon_{\mathrm{R}}+\varepsilon_{\mathrm{FR}}+\varepsilon_{v}^{f}-\varepsilon_{0}^{f}-\varepsilon_{v}^{i}+\varepsilon_{0}^{i}\right]^{2} / 4 E_{r} \\
\Delta G^{0}=G_{D H}^{0}-G_{A H}^{0}+e V
\end{gathered}
$$

Unlike redox-mediated electron tunneling ${ }^{57}$, the current is independent of the overpotential, eqs.(32) and (33). This is due to the character of eq.(21) as H-atom transfer between the neutral molecules $\mathrm{DH}$ and $\mathrm{AH}$. The proton tunneling factor is averaged over the intramolecular vibrations $^{4,5,12}$ but still depends strongly on the donor-acceptor molecular distance. Eqs.(26) and (29)-(33) describe the single-molecule charge transfer kinetics in the contact. Fig.8 shows representative current-bias voltage relationships. The current reaches constant values at large $V$ determined by the rate constant of the H-atom transfer step.

This approach follows concepts from condensed matter single-ET processes ${ }^{57}$. With the level of understanding and technology of the latter, implementation of the single-molecule PCET scheme suggested is likely to be within reach. 


\section{Some Conclusions and Outlooks}

PT and PCET have offered recent challenges in experimental innovation and theoretical framing. Hatom transfer and PCET in chemical and biological environments constitute a class where large KIEs and broad temperature ranges have opened for details such as heavy nuclear tunneling in gating modes and differential H/D distance effects. PT in double- or single-well proton environments with subtle interplay between the proton and solvent dynamics that lead to virtually complete erosion of the proton tunneling barrier is another increasingly better understood area. PT studies have been extended to the inverted free energy range ${ }^{39-41}$. Extension of the same basic theoretical framework to the electrochemical dihydrogen evolution reaction was introduced early ${ }^{7,66}$ and re-addressed in recent times with other theoretical tools added ${ }^{11}$. Less comprehensive exploitation of PT/PCET notions extends to areas such as:

- Coordination chemistry and catalysis. It was shown early ${ }^{67,68}$ that intramolecular $\beta$-hydride transfer, insertion and other elementary steps in industrially interesting condensed matter transition metal complex-based catalytic processes could be framed by PT/PCET/ $\mathrm{H}^{-}$-transfer theory as overviewed. Elementary PCET steps in ruthenium-polypyridine ${ }^{11,69}$ and rhenium-tyrosine complexes ${ }^{11,70}$ have been noted later to follow similar patterns.

- Along a related line, surface immobilized cuboidal $\mathrm{Mo}_{3} \mathrm{~S}_{4}$-cluster monolayers were recently found to be efficient catalysts in the electrochemical dihydrogen evolution reaction directly in fuel cell environment (carbon-based electrodes) $)^{71,72}$. The electrochemical studies were combined with single-molecule STM so that the catalytic efficiency per surface bound $\mathrm{Mo}_{3} \mathrm{~S}_{4}$-cluster molecule could be estimated. Viewed on a per molecule basis the catalytic efficiency was high and close to the maximum of the volcano plot of a broad range of metallic substrates. The elementary steps of the overall process involves PCET between the $\mathrm{Mo}_{3} \mathrm{~S}_{4}$-cluster and both $\mathrm{H}_{3} \mathrm{O}^{+}\left(\mathrm{H}_{5} \mathrm{O}_{2}{ }^{+}\right)$and the 
electrode with different intermediate Mo-oxidation states and $\mathrm{H}^{-} / \mathrm{H}$-atom coordination. Details of this sequence are not sorted out and would need comprehensive computational support.

Views on mechanisms of PT and EPC in water framed by phenomenological theory with computational support have been extended to proton conductance in polymer electrolyte membranes (PEM), Fig.9. These differ from bulk environments by the pore confinement of the proton transport and by the immobilized counter ions such as the $-\mathrm{SO}_{3}{ }^{-}$ions in $\mathrm{Nafion}{ }^{\circledR}$, as opposed to freely mobile counter ions in bulk proton conductivity ${ }^{26,52,53}$. The elementary PT act in the totally adiabatic limit of strong proton donor-acceptor interaction is in focus also in these environments. The bridge to observable proton conduction in the PEM environment of variable-range pore diameter and pore length, pore swelling on exposure to water environment, and overall threedimensional percolative proton transport patterns, however, prompt incorporation of other crucial elements in addition to the elementary PT process. These have been addressed in combination with PT theory but details are fraught with the absence of precise structural information about the PEM pore-confinement. The latter would have to include the following considerations ${ }^{26,52}$ :

- Distinction between "bulk" proton conductivity in the central pore region and $-\mathrm{SO}_{3}{ }^{-}-\mathrm{H}_{3} \mathrm{O}^{+}$ mediated proton conductivity along the pore walls.

- Estimation of the $\mathrm{H}^{+}$-concentration distribution across a given pore, $\rho(r)$ of diameter $\mathrm{R}$ and length $L_{\text {pore }}$, giving the single-pore conductance ${ }^{26}$

$$
G_{\text {pore }}=\frac{2 \pi}{L_{\text {pore }}} \int_{0}^{R} r d r \rho(r) \mu(r)
$$

where the mobility incorporates both surface and bulk proton conduction.

- Incorporation of pore swelling on exposure to aqueous solution.

- Extension of single-pore conductance to three-dimensional PEM pore networks with all the features of statistical pore distribution and swelling, combined with the fundamental PT process in these environments.

Fig.9

As further notes of observation, by the detailed structural information now available, broad temperature ranges addressed, and significant KIEs, the photocycle of the green fluorescent protein 


\section{Acknowledgement}

Financial support from the Danish Research Council for Technology and Production Sciences (Contract No. 274-07-0272) is acknowledged.

\section{References}

1. Bell, R.P. The Proton in Chemistry, 2nd Ed., 1973, Chapman and Hall, London, 1973.

2. Bell, R.P. The Tunnel Effect in Chemistry, 1980, Chapman and Hall, London.

3. Caldin, E.F.; Gold, V. Eds., Proton-Transfer Reactions, 1975, Chapman and Hall, London.

4. Kuznetsov, A.M., Charge Transfer in Physics, Chemistry and Biology, 1995, Gordon \& Breach, Reading.

5. Kuznetsov, A.M.; Ulstrup, J. Electron Transfer in Chemistry and Biology. An Introduction to the Theory, 1998; Wiley, Chichester. 
6. Dogonadze, R.R., Kuznetsov, A.M.; Levich, V.G., Elektrokhimiya 1967, 3, 739-742. Soviet Electrochemistry 1967, 3, 648-650.

7. Dogonadze, R.R.; Kuznetsov, A.M.; Levich, V.G. Electrochim. Acta 1968 , 13, 1025-1043.

8. Levich, V.G.; Dogonadze, R.R.; German, E.D.; Kuznetsov, A.M.; Kharkats, Yu.I. Electrochim. Acta, 1970, 15, 353-370.

9. Chapters in: Cohen, A.; Limbach, H.-H., Eds., Isotope Effects in Chemistry and Biology, 2006, CRC Press, Taylor \& Francis, Boca Raton.

10. Kuznetsov, A.M.; Ulstrup, J. Chem. Phys. 1994, 188, 131-141

11. Hammes-Schiffer, S.; Soudackov, A.V. J. Phys. Chem. B 2008, 112, 14108-14123.

12. Kuznetsov, A.M.; Ulstrup, J. Can J. Chem. 1999, 77, 1085-1096.

13. Kohen, A. and Klinman, J.P. Acc. Chem. Res. 1998, 31, 397-404.

14. Nagel, Z.D.; Klinman, J.P. Chem. Rev. 2006, 106, 3095-3118.

15. Kuznetsov, A.M.; Ulstrup, J. Ref. 9, Chapter 26, pp. 691-724.

16. a. Ulstrup, J.; Jortner, J. J. Chem. Phys. 1975, 63, 4358-4368. b. Søndergaard, N.C.; Ulstrup, J.; Jortner, J. Chem. Phys. 1976, 17, 417-422.

17. a. Brüniche-Olsen; Ulstrup, J. J. Chem. Soc. Faraday Trans. II 1978, 74, 1690-1701. b. J. Chem. Soc. Faraday Trans. II 1979, 75, 205-226.

18. a. German, E.D.; Kuznetsov, A.M.; Dogonadze, R.R. J. Chem. Soc. Faraday Trans. II 1980, 76, 1128-1146. b. German, E.D.; Kuznetsov, A.M. J. Chem. Soc. Faraday Trans. I 1981, 77, $397-$ 412. c. J. Chem. Soc. Faraday Trans. III 1981, 77, 2203-2212.

19. a. Wikstrom, M. Verkhovsky, M.I. Biochim. Biophys. Acta-Bioenergetics 2002, 1555, 128132. b. Kim, Y.C.; Wikström, M.; Hummer, G. Proc. Nat. Acad. Sci. USA 2007, 104, 21692174. c. Kim, Y.C.; Wikström, M.; Hummer, G. Proc. Nat. Acad. Sci. USA 2009, 106, 1370713712. d. Wikström, M. in: Hammerich, O.; Ulstrup, J., Eds., Bioinorganic Electrochemistry 2008, Springer-Verlag, Dordrecht, pp. 25-35.

20. Yoshikawa, S.; Muramoto, K.; Shinzawa-Itoh, K.; Biochim. Biophys. 2006, Aoyama, H.; Tsukihara, T.; Shimokata, K.; Katayama, Y.; Shimada, H. Biochim. Biophys. Acta 2006, 1757, 1110-1116.

21. Xu, J.C.; Voth G.A. Proc. Nat. Acad. Sci. USA 2005, 102, 6795-6800.

22. Xu, J.C.; Voth, G.A. Biochim. Biophys. Acta - Bioenergetics 2008, 1777, 196-201.

23. a. Silverman, D.N.; Lindskog, S. Acc. Chem. Res. 1988, 21, 30-36. b. Silverman, D.N.; McKenna, R. Acc. Chem. Res. 2007, 40, 669-675. 
24. Duda, D.M.; McKenna, R., in: Messerschmidt, A.; Huber, R.; Poulos, T.; Wieghardt, K., Handbook of Metalloproteins, Eds. 2004, Wiley, New York, Vol. 3, p. 1249-263

25. Maupin, C.M.; Mckenna, R.; Silverman, D.N.; Voth, G.A. J. Am. Chem. Soc. 2009, 131, 75987608.

26. Eikerling, M.; Kornyshev, A.A.; Kuznetsov, A.M.; Ulstrup, J.; Walbrand, S. J. Phys. Chem. 27. 2001, 105, 3646-3662.

27. van Thor, J.J.; Sage, J.T. Photochem. Photobiol. 2006, 5, 597-602.

28. van Thor, J.J. Chem. Soc. Rev. 2009, 38, 2935-2950.

29. Siebrand, W. and Wildman, T.A. Acc. Chem. Res. 1986, 19, 238-243.

30. a. Benderskij, V.A.; Gol'danskij, V.I.; Makarov, D.E. Phys. Rep. 1993, 233, 195-339. b. Benderskij, V.A.; Makarov, D.E.; Wight, C.A. Chemical Dynamics at Low Temperatures 1994, Wiley, New York.

31. Agmon, N. Chem. Phys. Lett. 1995, 244, 456-462.

32. a. Marx, D.; Tuckerman, M.E.; Hutter, J.; Parrinello, M. Nature 1999, 397, 601-604. b. Marx, D.; Tuckerman, M.E.; Parrinello, M. J. Phys. Condense Matter 2000, 12, A153-A159. c. Marx, D. ChemPhysChem 2007, 8, 209-210.

33. a. Day, T.J.F.; Schmitt, U.V.V.; Voth, G.A. J. Am. Chem. Soc. 2000, 122, 12017-12028. b. Izvekov, S.; Voth, G.A. J. Chem. Phys. 2002, 116, 10372-10376.

34. Kornyshev, A.A., Kuznetsov, A.M., Spohr, E. and Ulstrup, J.,J. Phys. Chem. B 2003, 107, 3351-3366.

35. Sumi, H.; Ulstrup, J. Biochim. Biophys. Acta 1988, 955, 26-42.

36. a. Cukier, R.I. J. Phys. Chem. 98 (1994) 2377-2381. b. R.I. Cukier, J. Phys. Chem. 100 (1996) 15428-15443. Cukier, R.I.; Nocera, D.G. Ann. Rev. Phys. Chem. 1998, 49, 337-369.

37. a. Kiefer, P.M. and Hynes, J.T. J. Phys. Chem. 2004, 108, 11793-11808. b. J. Phys. Chem. A 2004, 108, 11809-11818.

38. a. Kuznetsov, A.M.; Ulstrup, J. Russian J. Electrochem. 2004, 40, 1000-1009. b. Russian J. Electrochemistry 2004, 40, 1010-1018.

39. Andrieux, C.P.; Garnby, J.; Hapiot, P.; Savéant, J.-M. J. Am. Chem. Soc. 2003, 125, 1011910124.

40. a. Peters, K.S.; Kim, G.; J. Phys. Chem. A 2004, 108, 2598-2606. b. Heeb, L.R.; Peters, K.S. J. Phys. Chem. A 2006, 110, 6408-6414.

41. Peters, K.S. Acc. Chem. Res. 2009, 42, 89-96. 
42. Goguadze, N.G.; Hammerstad-Pedersen, J.M.; Khoshtariya, D.E.; Ulstrup, J. Eur. J. Biochem. 1991, 200, 423-429.

43. Farver, O.; Zhang, J.; Chi, Q.; Pecht, I.; Ulstrup, J. Proc. Nat. Acad. Sci. USA 2001, 98, 44264430.

44. Kuznetsov, A.M.; Medvedev, I.G.; Ulstrup, J. Electrochem. Comm. 2009, 11, 1170-1173.

45. Meyer, M.P. and Klinman, J.P. Chem. Phys. 2005, 319, 283-296.

46. Kubo, R.; Toyozawa, Y. Progr. Theor. Phys. 1955, 13, 160-182.

47. Kjær, A.M.; Ulstrup, J. J. Am. Chem. Soc. 1987, 109, 1934-1942.

48. Voth, G.A. Acc. Chem. Res. 2006, 39, 143-150. b. Paesani, F.; Voth, G.A. J. Phys. Chem. B 2009, 113, 5702-5719.

49. a. Markovitch, O.; Agmon, N. J. Phys. Chem. A 2007, 111, 2253-2256. b. Mol. Phys. 2008, 106, 485-495. c. Cox, M.J.; Timmer, R.L.A.; Bakker, H.J.; Park, S.; Agmon, N. J. Phys. Chem. A 2009, 113, 6599-6606.

50. a. Benoit, M.; Marx, D. ChemPhysChem 2005, 6, 1738-1741. b. Tuckerman, M.E.; Chandra, A.; Marx, D. Acc. Chem. Res. 2006, 39, 151-158.

51. Walbran, S; Kornyshev, A.A. J. Chem. Phys. 2001, 114, Art. No. 10039

52. a. Eikerling, M.; Kornyshev, A.A. J. Electroanal. Chem. 2001, 502, 1-14. b. J. Electroanal. Chem. 2002, 528, 196-197.

53. Petersen, M.K.; Voth, G.A. J. Phys. Chem. B 2006, 110, 18594-18600.

54. a. Eucken, A. Z. Elektrochem. 1948, 52, 6-24. b. Z. Elektrochem. 1948, 52, 255-269.

55. Gierer, A.; Wirtz, K. Ann. Phys. 1949, 6, 257-304.

56. Sumi, H. J. Phys. Chem. 1991, 95, 3334-3350.

57. Zhang, J; Kuznetsov, A.M.; Medvedev, I.G.; Q. Chi, Q.;Albrecht, T.;Jensen, P.S.; Ulstrup, J. Chem. Rev. 2008, 108, 2737-2791.

58. b. Kuznetsov, A.M.; Ulstrup, J. J. Phys. Chem. A 2000, 104, 11531-11540. b. Errata: J. Phys. Chem. A 2001, 105, 7494.

59. Faraday Discussions, Molecular Wires and Nanoscale Conductors 2006, 131, 5-416.

60. Aviram, A. J. Mol. Electronics 1988, 4, S99-S104.

61. Barbara, P.F.; Trommsdorff, H.P. Eds., Chem. Phys. 1989, 136, 53-360, Special issue on Spectroscopy and dynamics of elementary proton transfer in polyatomic systems.

62. a. Guallar, V.; Batista, V.S.; Miller, W.H. J. Chem. Phys. 1999, 110, 9922-9936. b. J. Chem. Phys. 2000, 113, 9510-9522. 
63. A.M. Kuznetsov; J. Ulstrup, Elektrokhimiya 2003, 39, 9-15.

64. a. Kuznetsov, A.M.; Medvedev, I.G.; Ulstrup J. J. Chem. Phys. 2007, 127, Art. No. 104708. b. Russian. J. Electrochem. 2008, 44, 983-991.

65. Albrecht, T.; Guckian, A.; Kuznetsov, A.M.; Vos, J.G.; Ulstrup, J. J. Am. Chem. Soc. 2006, $128,17132-17138$.

66. Kharkats, Yu.I.; Ulstrup, J. J. Electroanal. Chem. 1975, 65, 555-572.

67. Søgaard Andersen, P.; Ulstrup, J. Acta Chem. Scand. 1983, 37, 585-593.

68. Creutz, C.; Sutin, N. J. Am. Chem. Soc. 1988, 110, 2418-2427.

69. Iordanova, N.; Hammes-Schiffer, S. J. Am. Chem. Soc. 2002, 124, 4848-4856.

70. Ishikita, H.; Soudackov, A.V.; Hammes-Schiffer, S. J. Am. Chem. 2007, 129, 11146-11152.

71. Kristensen, J.; Zhang, J.; Ulstrup, J.; Chorkendorff, I.; Ooi, B.L. Dalton Trans. 2006, 39853990.

72. Jaramillo, T.F.; Bonde, J.; Zhang, J.; Ooi, B.L.; Andersson, K.; Ulstrup, J.; Chorkendorff, I. J. Phys. Chem. C 2008, 112, 17492-17498.

\section{Legends for Figures}

Fig.1

Double-well potential free energy projections on the proton coordinate, $r_{p}$, along the environmental coordinate(s) $\left\{q_{k}\left(r_{p}\right)\right\}$. The proton is trapped near the donor and near the acceptor, when $\left\{q_{k}\left(r_{p}\right)\right\}$ takes its equilibrium values in the reactants', $\left\{q_{\kappa D O}\left(r_{p}\right)\right\}$, and products' state, $\left\{q_{\kappa A O}\left(r_{p}\right)\right\}$, respectively.

Fig.2

Potential free energy projections along the proton coordinate, $r_{p}$ at the reactants, $\left\{q_{\kappa D O}\left(r_{p}\right)\right\}$ and products' equilibrium states, $\left\{q_{\kappa A O}\left(r_{p}\right)\right\}$, and in the transition state $\left\{q_{\kappa}^{*}\left(r_{p}\right)\right\}$, all with respect to the environmental solvent nuclear coordinates.

Fig.3

Left: Comparison of nuclear tunnelling barrier at given height in the "normal" and inverted free energy range. The latter barrier is narrower and tunneling much more facile than the former.

Right: The potential surface splitting in the inverted free energy region. The stronger the splitting the less likely is the transition from the reactants' (upper) to the product's (lower) state. This is opposite to the "normal" free energy region. 
Fig.4

Electron-proton potential free energy surfaces, $U_{v w}^{e l-p r o t}\left(\left\{q_{\kappa}\right\}\right)$ to show the effect of vibrational excitation in high-frequency local modes such as proton stretching and bending modes in the "normal" and inverted free energy regions, in the partially or totally diabatic limits. The surfaces represent a solvent reorganization free energy of $0.9 \mathrm{eV}$ and the products' surfaces are separated by the vibrational energy of the unperturbed proton stretching mode, $0.3 \mathrm{eV}$. Local vibrational excitation in the former region raises the activation free energy. In contrast, PT to excited vibrational states in the products' (electronic) state lowers the activation free energy and gains importance when the driving force exceeds the combined solvent reorganization free energy and local mode vibrational energy, $\left|\Delta G^{0}\right|>E_{r}+\hbar \Omega_{p}$.

\section{Fig.5}

A: "Generic" PT mode in the Grotthuss mechanism of EPC. The solvent molecule $\mathrm{S}_{\mathrm{D}}$ is initially hydrogen-bonded to the proton acceptor, $A$, while the solvent molecule $S_{A}$ near the proton donor $D$ is less strongly bonded. PT is triggered by synchronous displacement and hydrogen bond formation and breaking.

B: Bottom: Double-PT via the Zundel complex $\mathrm{H}_{5} \mathrm{O}_{2}{ }^{+}$. The proton is initially located symmetrically between the water molecules 1 and 2 . In the final state the proton has been translocated to a symmetrical position between the water molecules 2 and 3. Mechanisms analogous to these are expected to operate in proton conduction through biological and synthetic membranes.

\section{Fig.6}

PT in a single-well potential at the initial equilibrium ( $\boldsymbol{s}_{\boldsymbol{D} \boldsymbol{0}}$ and transition configuration ( $\mathrm{s}^{*}$ ) of a lowfrequency gating or other mode. The proton remains in its ground vibrational state throughout the reaction. $\varepsilon_{p}$ is counted from the bottom of the potential well.

\section{Fig.7}

Free energy plot into the inverted region. The solvent reorganization free energy is $0.9 \mathrm{eV}$ and the proton vibrational energy $\hbar \Omega_{H}=0.3 \mathrm{eV}\left(\Omega_{H} \cong 3000 \mathrm{~cm}^{-1}\right)$. The fully drawn line corresponds to a 
PT distance, $\Delta r_{H}^{*}=0.3 \AA$, the dashed line to $\Delta r_{H}^{*}=0.2 \AA$ (coupling constants $\frac{1}{2}\left(m \Omega_{H} / \hbar\right)\left(\Delta r_{H}^{*}\right)^{2}=$ 5 and 2 , respectively).

Fig.8

Left: A scheme of concerted single-proton relay. The first step is proton-coupled two-ET, the second step H-atom transfer in the opposite direction (dashed line).

Right: Dependence of the normalized current on the bias voltage for concerted ET/PT in the partially adiabatic limit. PT vibrational ground states only. The H-atom transfer reaction is considered to be activationless, i.e. $G_{A H}^{0}-G_{D H}^{0}=E_{r}$ with $E_{r}=5 k_{\mathrm{B}} T$. The current is normalized to the pre-exponential factor of the PT rate constant. The curves represent different ratios of the preexponential factors of PT and H-atom transfer, $c$, i.e.: $1: c=0.1 ; 2: 0.05 ; 3: 0.01$.

Fig.9

Proton conductivity in Nafion ${ }^{\circledR}$ PEM pore, schematic. Approximate representative distances between $-\mathrm{SO}_{3}{ }^{-}$and $\mathrm{H}_{3} \mathrm{O}^{+} / \mathrm{H}_{2} \mathrm{O}$ proton donor and acceptor groups are shown. 


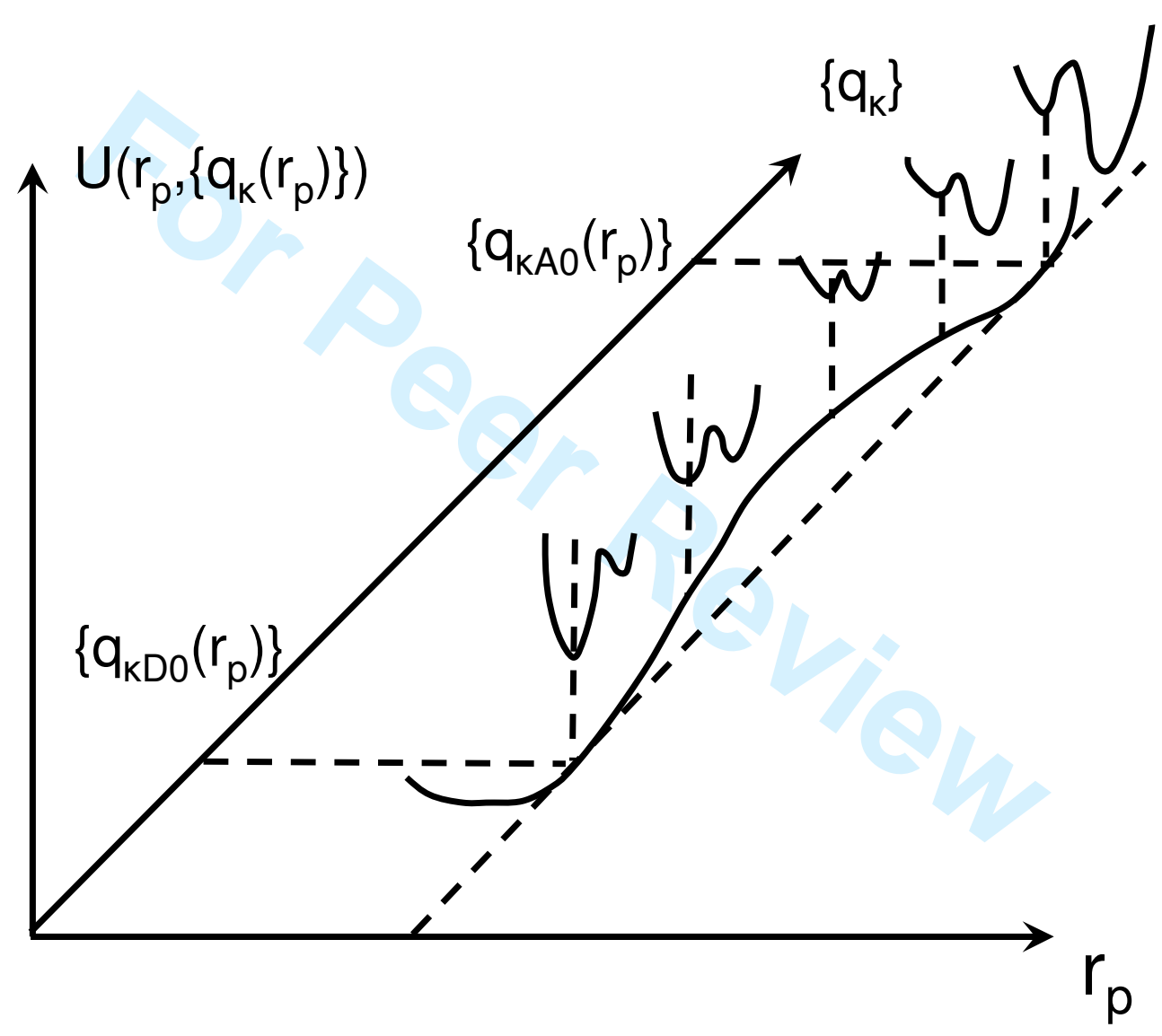

Figure 1 

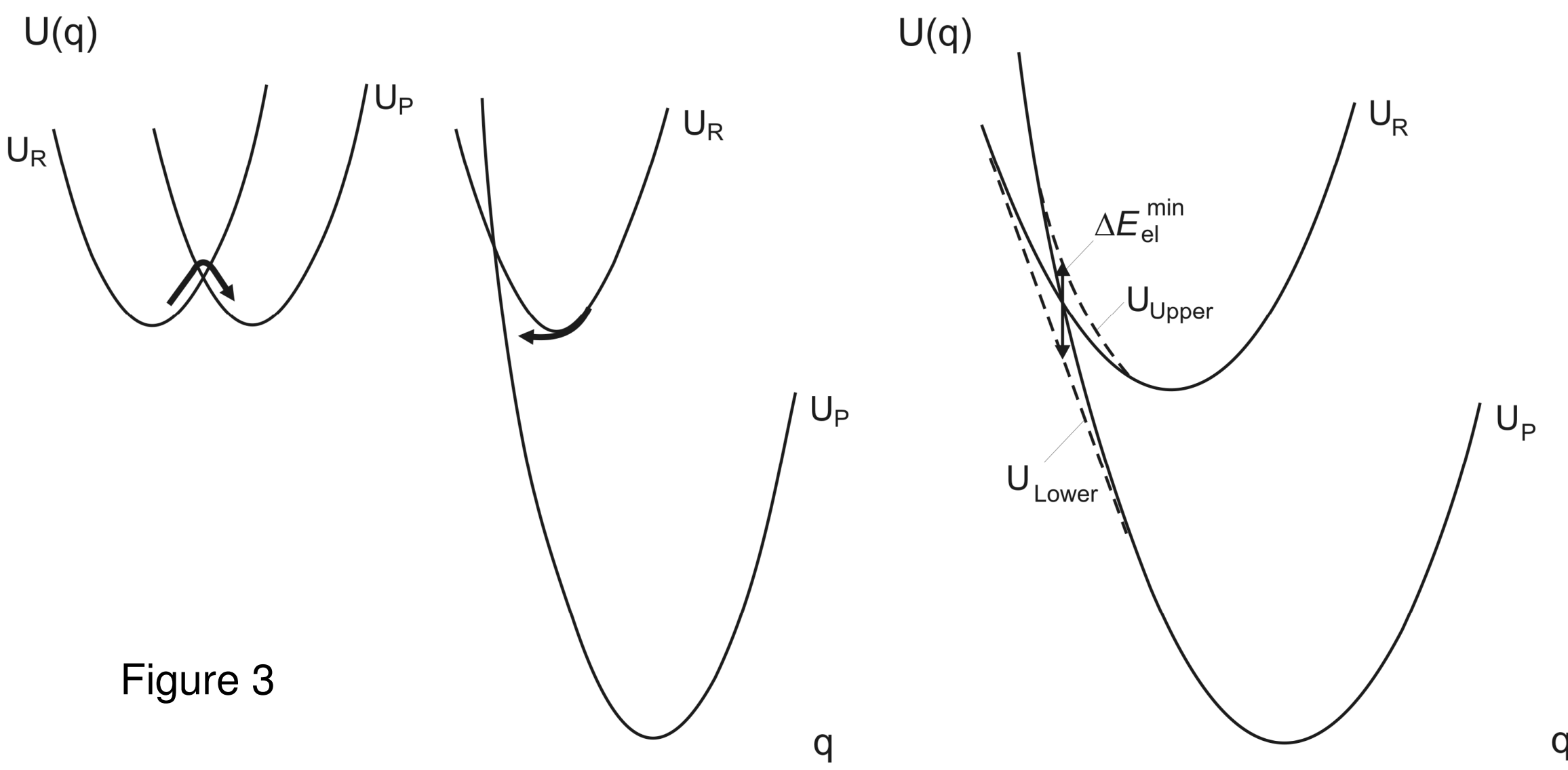

http://mc.manuscriptcentral.com/poc 


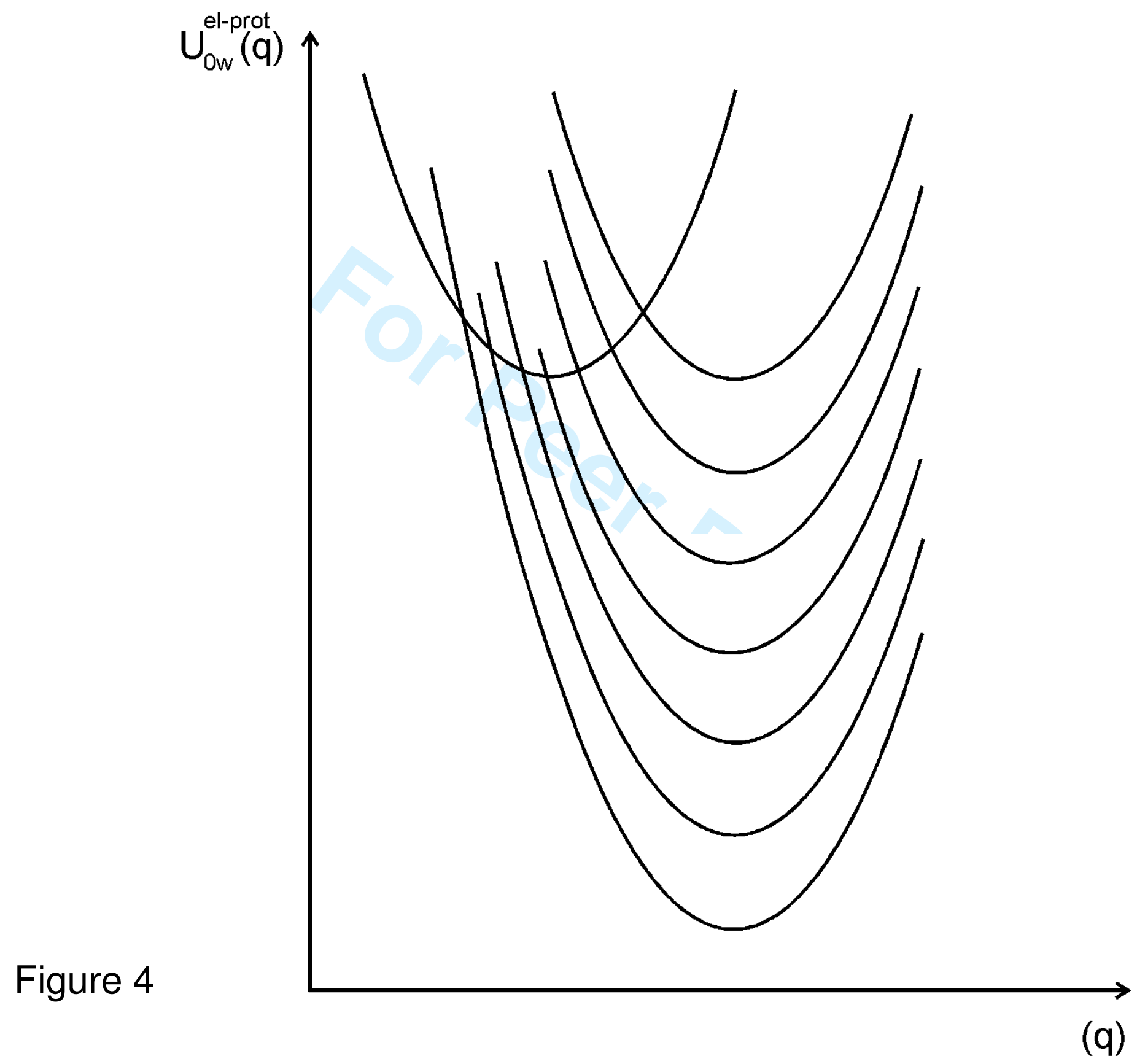

http://mc.manuscriptcentral.com/poc 


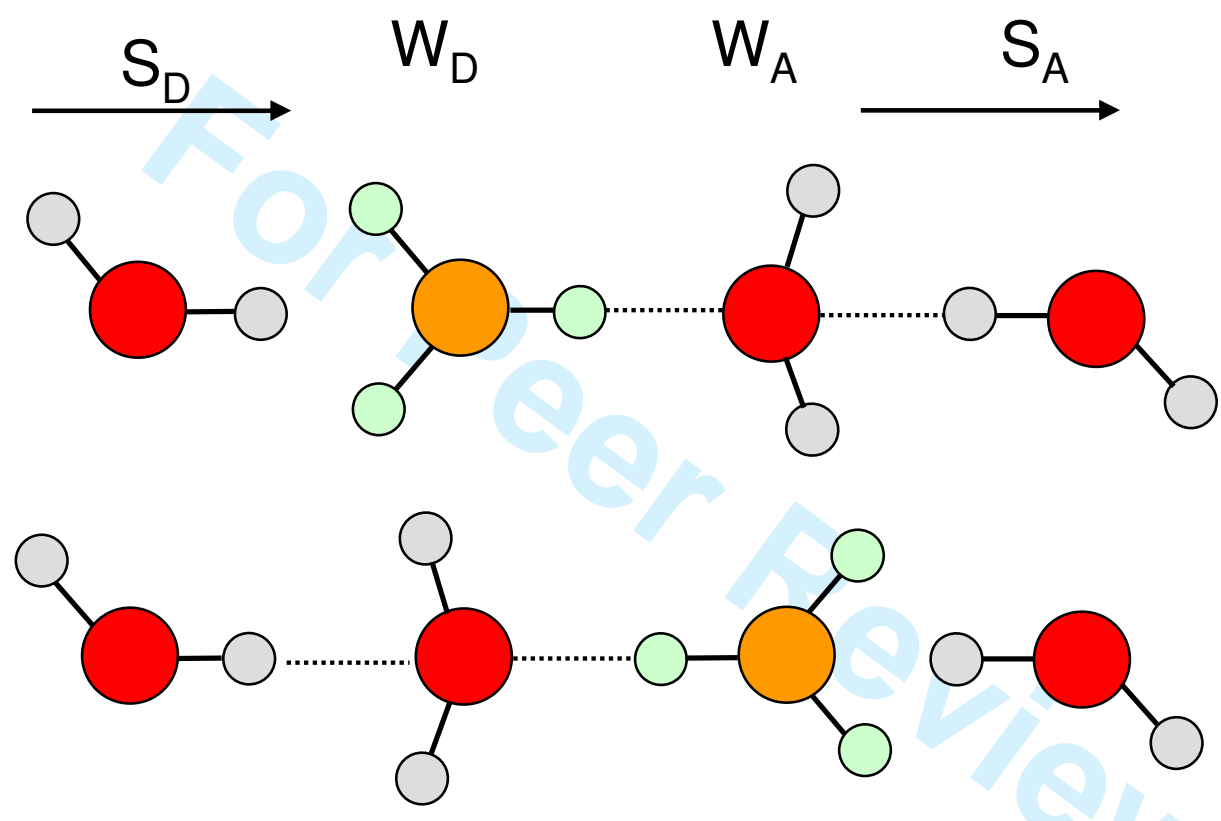

Fig.5A 


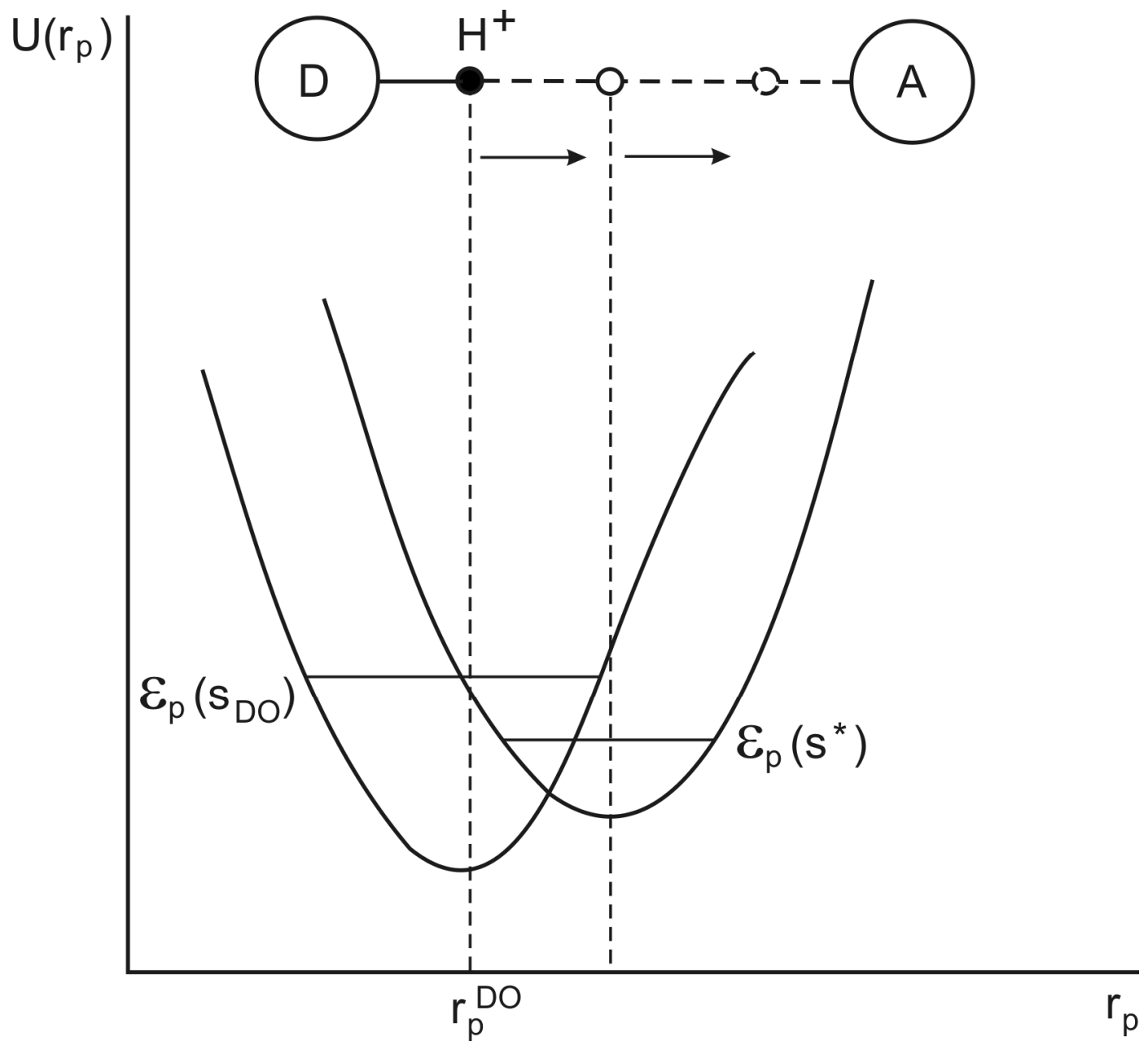

Figure 6 


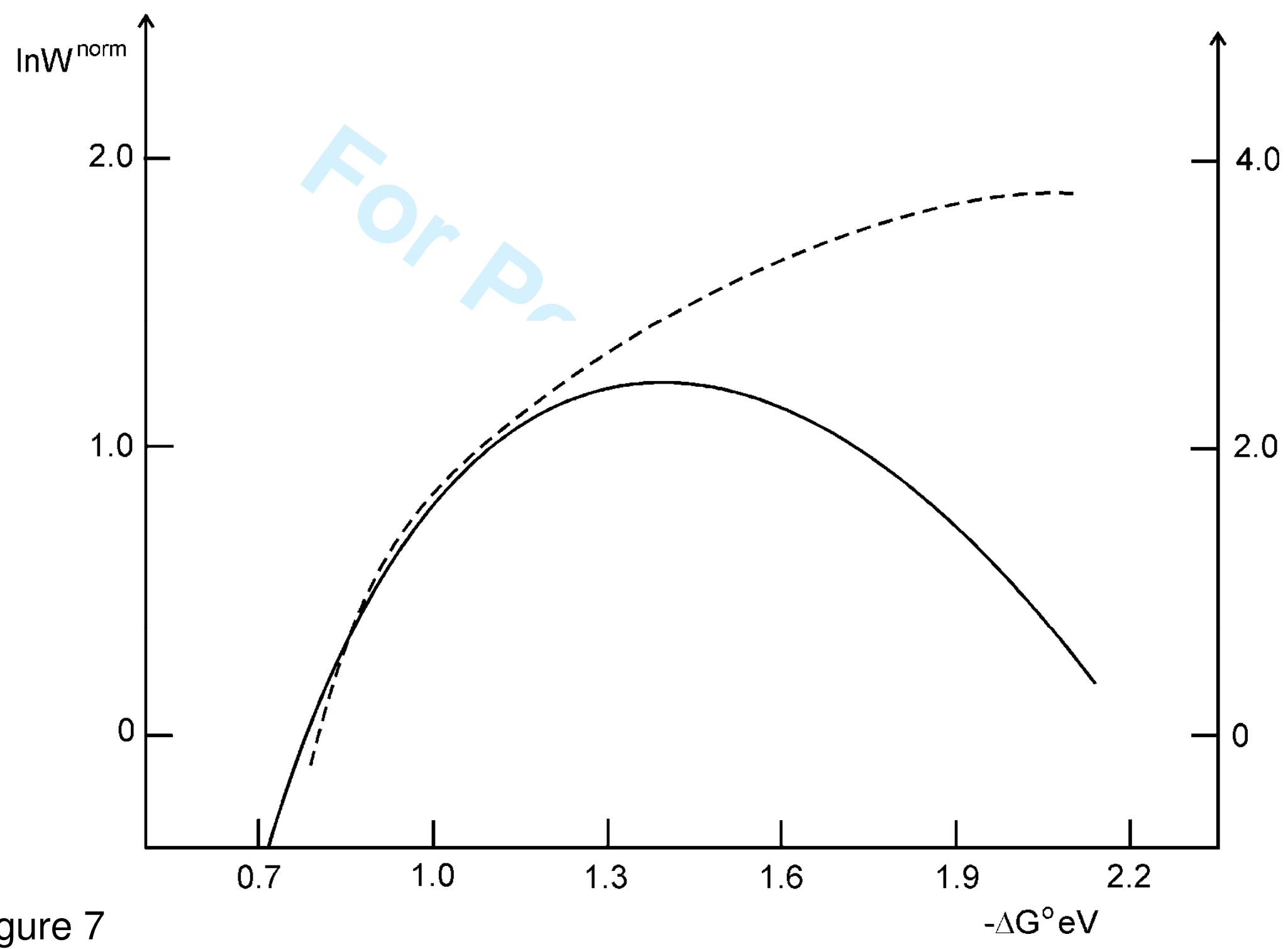

Figure 7 

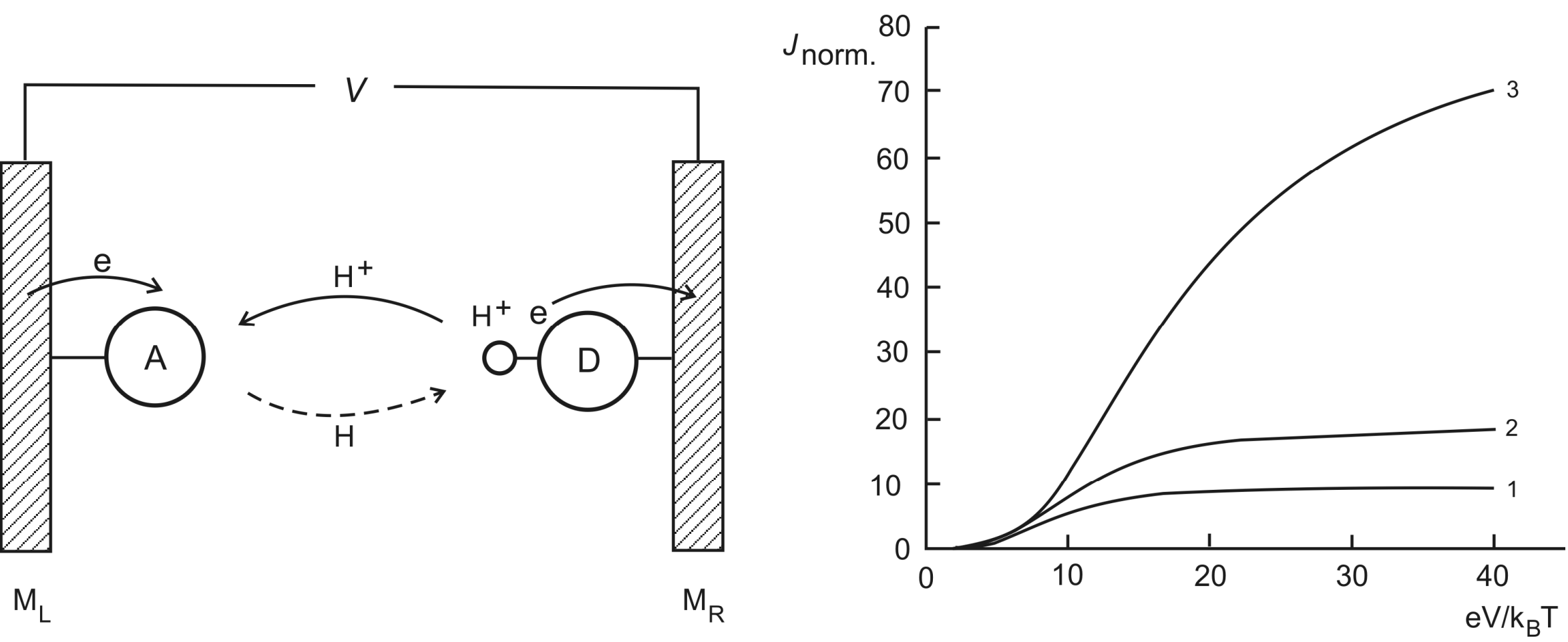

Figure 8 
